\title{
Comprehensive Equipment Management System Based on Web Service
}

\author{
Peng Li \\ Department of Research and Development \\ Wuxi CAS Ubiquitous Information Technology R\&D \\ Center CO., LTD \\ Wuxi, China \\ pengli@sia.cn
}

\author{
Mingrui Shi \\ Department of Control Science and Engineering \\ Zhejiang University \\ Hangzhou, China
}

\begin{abstract}
A comprehensive equipment management system based on web service is introduced, and design idea is described from the perspective of function scope, main functions and system integration. The key technologies of foreground model and background services are described. The system is integrated in web service based on integration capability and extension ability, which can be integrated in manufacturing execution system, and information management of equipment can be executed rapidly.
\end{abstract}

Keywords-Equipment Management Maintenance Repair Web Service

\section{INTRODUCTION}

Equipment management system emphasizes systematic, scientific and standardized management, applying a series of theories \& methods and using a series of technological, economic and organizational measures to have a scientific management on matter \& value movement morphology of equipment during operation \& maintenance following inproduction use, maintenance, repair, modification, update and scrap $^{[1]}$.We summarize experience in relation to equipment using, maintenance, repairing \& cost control, adapt to new demand, make a scientific and overall planning on equipment resources and attempt to improve comprehensive using efficiency \& benefits to realize requirements of green production and guarantee corporate economic stable operation and perfect equipment management system, quicken equipment management informatization, strengthen equipment management standardization, put TPM field standardization management, regulate equipment management working process to improve efficiency of equipment, guarantee safe and reliable operation of equipment and lay the solid foundation for corporate all-round development ${ }^{[2-3]}$.

In terms of equipment management inside an enterprise, there are often several shortages as below ${ }^{[4-6]}$ :

(1) Internal enterprise equipment management is mainly based on traditional management by experience. Equipment management becomes relatively lagging by recording cycle and designating maintenance work order manually and stays at daily maintenance and failure repair relying on subjective experience.

(2) Equipment management is lack of a unified evaluation standard. Some indictors still use off-line manual way of statistics. Because of large data volume, data statistics is lagging even with personal errors.

(3) Although internal enterprise has implemented information system, such as: MES, ERP, WMS, etc., there are still information island in the aspect of equipment management because of relatively lower equipment management informatization application level.

(4) It makes equipment operation and maintenance more difficult and equipment maintenance strategy determination more complex to have a large amount of higher-automated complex processing equipments, therefore, every equipment operation and maintenance mode needs to be determined through a detailed operation and maintenance history record. Daily maintenance, routing inspection, real-time dynamic monitoring and failure maintenance record are the basis of making maintenance contents and time. Only through constant analysis on equipment operation state and failure cause, can equipment maintenance process be optimized on this basis.

(5) Past equipment maintenance and repair experience is an extremely valuable intellectual property for enterprises. Once technicians who maintains and manages original equipments quit their jobs, equipment maintenance specialists' knowledge won’t be learnt.

\section{SYSTEM FUNCTION DELIMITING}

Equipment operation and maintenance management system aims at corporate production equipments and involves in relevant field production equipments. System controls and analyzes equipments during operation and maintenance from in-production use, maintenance, repair, modification, update and scrap. System management focuses on dynamic management, manages all various working factors in relation to equipments, organizational system of equipments, equipment operating and maintaining records, failure accidents, spot check, maintenance, repair, modification, update, scrap, equipment lubrication, spare parts, special equipments, organizations and uses technical methods like remote video maintenance and audio/video specialists' knowledge management to standardize equipment management and serviceman's training and so on into an optimized management system, build high-efficient technical records during equipment operating \& maintaining and ensure normal operation of equipments. 


\section{SYSTEM FUNCTION INTRODUCTION}

Equipment operation and maintenance management system aims at equipments used for production operation in workshop and makes an all-round management on a series of links of operation, failure, maintenance, cost and so on during the process from in-production use to scrap. It utilizes real-time data acquisition system or manual informational input to record and save equipment data during operation and maintenance and, on a basis of this, builds multi-level equipment-relevant comprehensive indicator evaluation system from factory, workshop to equipment through abundant graphical analysis and powerful report presentation to provide advanced integrated solution, improve equipment management technology \& management level and give basis \& guarantee for corporate decision making.

Under demand analysis, equipment operation and maintenance management system has main functions as shown in the Figure 1.

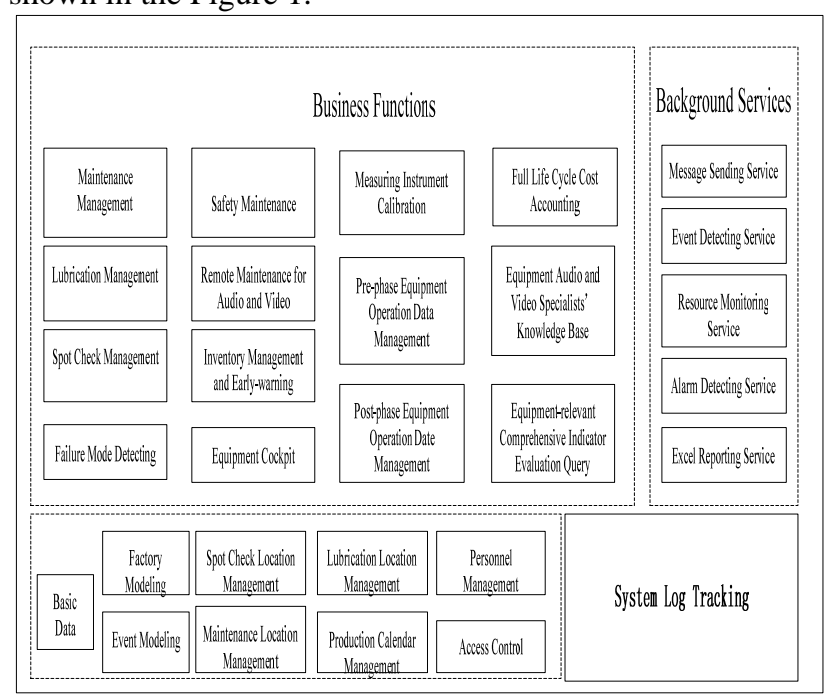

Figure 1. System Function Diagram

\section{A. Basic Data}

(1) Factory Modeling: It uses level-by-level navigation, layer-by-layer detailed way and a visualized method to build multi-level equipments following factory, workshop, production line, equipment and acquisition point. It covers some basic information in relation to equipments and lays a solid foundation for integration of equipment management. It refers to existing equipment coding specification to build a unified coding system on production equipments and spare parts in accordance with different equipment type, equipment class and spare part type, configures equipment data including equipment productivity, production line \& equipment in-production time, etc. and manages \& maintains basic data including technical data, drawing, etc..

(2) Event Modeling: It aims at equipments, defines the relevant modeling for production events (such as: work start \& stop, etc.) for key process and reflects actual production activity at production field. In the event of emerging issues, it will inform relevant subscriber and show in client side automatically $^{[7]}$.

(3) Maintenance Location Management: It decomposes equipments in accordance with maintenance business demand and, for different maintenance locations, designates different maintenance strategies including maintenance cycle, maintainer, maintenance and replacement part list, spare part alternatives, maintenance standard, maintenance documents, maintenance audio and video, etc.

(4) Spot Check Location Management: It decomposes equipments in accordance with spot check business demand and, for different spot check locations, designates different spot check strategies including spot check cycle, spot checker, spot check standard, spot check documents, spot check audio \& video, etc ${ }^{[8]}$.

(5) Lubrication Location Management: It decomposes equipments in accordance with lubrication business demand and, for different lubrication locations, designates different lubrication strategies including lubrication cycle, lubricant brand, lubricating consumptive quotas, lubrication standard, lubrication documents, lubrication audio and video, etc.

(6) Production Calendar Management: It can make relevant schedule for planned production, legal holidays, planned production stop and so on in accordance with different levels, such as: factory, department, production area, production equipment, etc. and provide basis and reference for making plans, such as: maintenance, spot check, lubrication, etc.

(7) Personnel Management: It can control adding, deleting, searching and modifying functions of involved personnel's information including name, employee number, phone number, employee's two-dimension code, department, shift and group, position, login password, etc.

(8) Access Control: It can be set up in detail for whether authorized officer has the right to access specific functional menu. In addition, it is allowed to limit different-level personnel to use and make them maintain different equipments in accordance with their technical proficiency.

\section{B. Background Service}

1) Test Message Sending Service: It packages API inside GSM MODEM in a way of Web Service and provides a unified invocation pattern, monitor received test message \& have a relevant analysis for modules that need to send text message.

2) Event Detecting Service: It has a real-time monitoring on occurred events in accordance with event model. Event trigger can be sent to event detecting service through message bus. It can also monitor designated parameters through reading and writing service. When parameters are changed in accordance with trigger condition, event handler is called.

3) Resource Monitoring Service: It is responsible to make a dynamic monitoring on resource-monitoring-modeldefined equipment resource state, parameter, etc. and publish relevant information to subscriber. 
4) Alarm Detecting Service: It has a real-time monitoring on alarming parameter in accordance with alarm model. When parameters are changed in accordance trigger conditions, alarming information occurs. Or a program or stored process is implemented every once in a while. Conditions under which alarming information occurs are predefined in program or during stored process. When conditions are met, alarming information occurs.

5) Excel Reporting Service: It imports data into Excel template based on automated function of Excel and utilizes VBA function of Excel to realize visualized representation of data after data is got from database.

\section{Business Function}

1) Equipment Maintenance and Repair Management: It makes and issues cyclic repair plan, heavy repair plan and preventive maintenance plan in accordance with equipment failures, required maintenance time and information from preventive maintenance.

Heavy Repair Plan Management for Equipments: It includes heavy repair plan making \& decomposing, issuing, implementing, reviewing, etc.

Preventive Maintenance Plan Management for Equipments: It includes preventive maintenance plan making, issuing, implementing \& reviewing, statistical query on its implemented process, etc.

Post Maintenance Plan Management for Equipments: It includes post maintenance plan making, issuing, implementing \& reviewing and statistical query on its implemented process.

2) Lubrication Management: As is shown in Figure 2, it is used for information guide and maintenance on daily lubrication for equipments, aiming to standardize equipment lubrication management and improve lubrication quality and efficiency, including lubrication plan making, issuing, implementing \& reviewing, statistical query on its implemented process, etc.

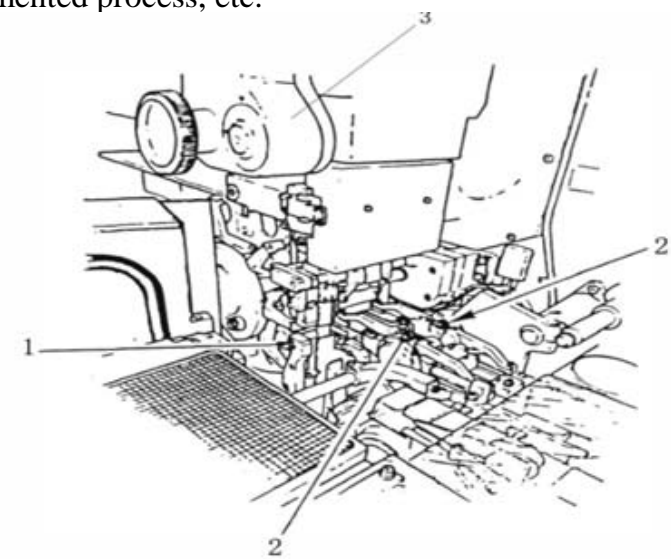

Main Driving Wheel Axle of Tinffoil Acceler ation Roller High Temperature Grase 7014 $10 x 1$ mont

Front and Back Ends of Whed No 1 High Tenperature Grease7014-10r 3 months

Alum inized paper Hend-plate Wheel Garbox High Temperatre Grease7014-10x 1 year

Figure 2. Knowledge Base
3) Spot Check Management: It is used for information guide on daily spot check for equipments, aiming at standardize spot check management for equipments and improve spot check quality and efficiency, including spot check plan making, issuing, implementing \& reviewing, statistical query on its implemented process, etc.

4) Safety Maintenance Management: It is used to make equipment stopping and locking function used in maintenance, spot check and lubrication to maximize eliminating hidden dangers during the process of implementing relevant business, such as: injury and even death caused by operators' mis-starting equipments.

5) Inventory Management and Early-warning: It is used for different spare parts to build basic information base including their name, picture, location, two-dimension code, safety inventory level, supplier, etc. and, to avoid delaying relevant work due to insufficient spare parts, make an early warning in accordance with future replacement plan consumption, in-transit information, safety inventory, etc.

6) Equipment Cockpit: It displays all relevant information based on equipments, such as: current state, next maintenance time, next lubrication time, next spot check time, spare part replacement data, spot check data, lubrication data, maintenance data, currently-processed product name, lot number, etc.

7) Measuring Instrument Calibration Management: It is used to support secondary development and make a selfdefined on inaccurate early-warning rules and modifier formulas to maximize reducing measurement errors by way of soft computing.

8) Equipment Audio and Video Specialists’ Knowledge Base: It makes specialists' experience during maintenance, spot check and lubrication kept in a way of audio and video and correlates it to relevant equipments. It is regarded as operation standard to make relevant activities during the process of implementation and executed in accordance with standard process.

9) Equipment-relevant Comprehensive Indicator Evaluation System: According to different analysis and evaluation angles, it is divided into production indicator analysis, energy consumption indicator analysis, equipment indicator analysis, etc. It includes equipment utilization rate, equipment serviceability rate, effective operation rate, equipment breakdown rate, OEE \& TEEP and makes an allround analysis on influence of manufacturing personnel, equipments, raw materials, methods and environment on equipment efficiency and its root cause[9].

\section{System Function}

1) Data Archiving and Backup: It has data archiving and backup automatically for historical data in different data sheet according to a certain cycle to prevent from long-time waiting for data query caused by sharp increase of data volume. 
2) System Log Recording and Tracking Management: It mainly records operators' deletion and modification records on important information including plan, equipment, etc. and also operators' time for shift change, system user login time, etc. and tracks relevant historical operational records through multiple ways of employee number, IP, operating time and so on to have a backward tracking on emerging issues.

\section{SYSTEM INTEGRATION}

Equipment management starts from equipment inproduction use till scrap and goes through the whole life cycle during operation and maintenance. Therefore, there are so many involved departments to be considered, which requests that it needs to read data from the system in ERP, such as: equipment purchasing, equipment standing book, etc. and integrate with production equipments in workshop to get various parameters in relation to equipments and realize an all-round management on production equipments. Relevant interfaces are shown in Figure 3.

(1) Data Acquisition Interface

System needs to make a real-time monitoring on state information of managed equipments that can be extracted from real-time historical database which can get state information in relation to equipments from equipment control system (such as: DCS, etc.) in accordance with OPC, etc.

For equipment state information that does not support automatic acquisition temporarily, this system provides manual input and reserves data acquisition interface. In case that such equipment state information supports automatic acquisition in the future, this system can realize automatic acquisition of equipment state by configuration.

(2) Third-party Software Interface

This system and the third-party software use the way to call Web Service interface for communication.

(3) Integration with ERP System

This system needs to have a data and application integration with the system in ERP, such as: equipment purchasing, equipment standing book management, cost management, human resource management, etc. (For spare parts, purchasing application is controlled by safety inventory).

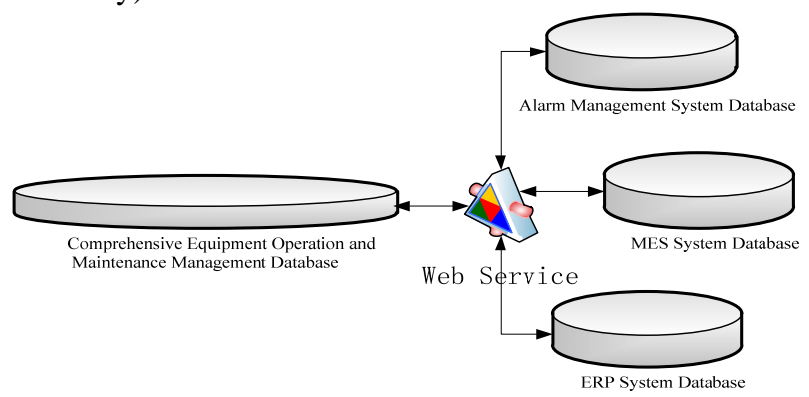

Figure 3. System Integration Frameworks

\section{V.CONCLUSION}

This paper integrates actual demand for equipment management to make a detailed description on system function delimiting, main system functions and demand analysis \& overall structure based on software system, brings up the overall solution for equipment-relevant comprehensive management system based on Web Service, introduces basic data (including system-inside factory model, event model, etc.), business function (including equipment spot check, lubrication, maintenance, etc.) and background service (including event, alarming, resource monitoring, etc.) and gives finally KPI evaluation system based on OEE, breakdown rate, effective operation rate and so on to digitalize the overall business process of equipment management and realize closed-loop feedback of management process. System has a good configurability to build an overall solution for equipment-relevant comprehensive management in accordance with clients' demand and integratability to make system efficient to solve "Information Island" and utilize original basic systematic data to reduce maintenance workload of relevant data.

\section{REFERENCES}

[1] Jiangrong. Application of ERP System in Equipment Management [J]. Construction Machinery and Maintenance, 2013, 1: 148 149.

[2] Li Zhencheng, Zheng Jianfeng. Application of Spot Check and Monitoring System in Equipment Management of Coal-dressing Enterprises [J]. Public Communication of Science and Technology, 2013, 1: 140 142.

[3] Su Hongsheng. Preventive Maintenance and Control Strategy for Repairable Parts [J]. Journal of Zhejiang University (Engineering Science), 2010, 7: 1308-1314.

[4] Jiang yongrui, Chang Xiuqun, Jie Jinliang. Development and Application of Cigarette-making Equipment Management System Based on B/S Mode [J]. Tobacco Science and Technology, 2008, 11: 27-29.

[5] Tangbo, Deng Changzheng, Wanggang. Research and Application of Preventive Maintenance of Power Station Equipment in EAM System. Electric Power Press, 2009, 24(5): 370-374.

[6] Lipeng, Shi Haibo, Shang Wenli. Factory Model Design and Modeling Method Research Based on SP95 Standard [J]. Computer Integrated Manufacturing Systems, 2009, 15 (3):458-462.

[7] Pan F C, Peng H, Shi H B. Event-based Production Process Traceability Model [C], Proceedings of the World Congress on Intelligent Control and Automation, 2006, 6: 1217-1232.

[8] Meng Xiangting, Guo Daquan. Tobacco Equipment Spot Check System Based on .NET [J] Microcomputer Information, 2011, 27(1):156-158.

[9] Lipeng, Shi Haibo, Shang Wenli. Design \& Implementation of Equipment-relevant Comprehensive KPI System [J]. Computer Engineering and Applications. 2011, 47(22):26-29. 